\title{
Valoración del Riesgo Cardiovascular en Varones Conductores Profesionales del Area Mediterránea Española y Variables Asociadas
}

\author{
ASSESSMENT OF CARDIOVASCULAR RISK IN MALE PROFESSIONAL DRIVERS OF THE SPANISH \\ MEDITERRANEAN AREA AND ASSOCIATED VARIABLES
}

\begin{abstract}
Ángel Arturo López González,2, María Gil Llinás', Milagros Queimadelos Carmona ${ }^{3}$ Irene Campos González4, Pilar Estades Janer¹, Rosa González Casquero²

1. Servicio de Prevención de Riesgo Laborales. Servei Salut Illes Balears. Hospital Son Llatzer. Palma de Mallorca. España.

2. Servicio de Prevención de Riesgo Laborales. Servei Salut Illes Balears. Hospital de Inca. España.

3. Servicio de Prevención de la Enfermedad. Dirección General de Salud Pública y Participación. Govern de les Illes Balears. España.

4. Servicio de Radiología. Hospital Son Llatzer. Palma de Mallorca. España.
\end{abstract}

\section{RESUMEN}

Introducción. El riesgo cardiovascular es una de las principales preocupaciones de los servicios de salud laboral.

Material y métodos. Se realiza un estudio retrospectivo y transversal en 2944 varones conductores profesionales (487 taxistas, 908 conductores de autobús y 1549 conductores de camión) y se valoran diferentes variables relacionadas con riesgo cardiovascular.

Resultados. El 27,4\% de los conductores son obesos, el 25\% hipertensos, un $48 \%$ presentan valores elevados de colesterol y un $29,5 \%$ triglicéridos altos, mientras la hiperglucemia aparece en el 6,5\%. Un 15,8\% cumplen los criterios de síndrome metabólico, un 2,6\% presentan valores elevados en la escala REGICOR y un 7,9\% en la escala SCORE. El riesgo elevado de padecer diabetes tipo 2 con la escala Findrisk aparece en el 13\% de los conductores.

Conclusiones. Globalmente, el colectivo de taxistas es el que presenta peores resultados en todas las variables relacionadas con riesgo cardiovascular.

(López A, Llinás M, Queimadelos M, Campos I, Estades P, González R, 2018. Valoración del Riesgo Cardiovascular en Varones Conductores Profesionales del Área Mediterránea Española y Variables Asociadas. Cienc Trab. Ene-Abr; 20 [61]: 1-6).

Palabras clave: RIESGO CARDIOVASCULAR, CONDUCTOR PROFESIONAL, HÁBITOS SALUDABLES.

\section{ABSTRACT}

Introduction. Cardiovascular risk is one of the main concerns of occupational health services.

Material and methods. A retrospective and cross-sectional study was conducted in 2944 professional male drivers (487 taxi drivers, 908 bus drivers and 1549 truck drivers) and different variables related to cardiovascular risk were assessed.

Results. $27.4 \%$ of the drivers are obese, $25 \%$ hypertensive, $48 \%$ have high cholesterol and 29.5\% high triglycerides, while hyperglycemia appears in 6.5\%. 15.8\% met the metabolic syndrome criteria, $2.6 \%$ presented high values on the REGICOR scale and 7.9\% on the SCORE scale. The high risk of type 2 diabetes with the Findrisk scale appears in $13 \%$ of drivers.

Conclusions. Globally the group of taxi drivers is the one that presents worse results in all the variables related to cardiovascular risk.

Key words: CARDIOVASCULAR RISK, PROFESIONAL DRIVER, HEALTHY HABITS.

\section{INTRODUCCIÓN}

Actualmente asistimos a un rápido incremento de las enfermedades crónicas no transmisibles, entre ellas las enfermedades cardiovasculares (ECV). Las ECV son la primera causa de muerte en el mundo y guardan una estrecha relación con diferentes factores de riesgo modificables a través de un cambio en el estilo de vida de las personas. De aquí surge la necesidad de desarrollar estrategias de

\section{Correspondencia / Correspondence:}

Ángel Arturo López González

Servicio de Prevención de Riesgo Laborales, Servei Salut Illes Balears Hospital Son Llatzer

e-mail: angelarturo.lopezgonzalez@hsll.es

Recibido: 06 de noviembre de 2017 / Aceptado: 12 de diciembre de 2017 prevención y detección precoz de aquellos grupos de población que se consideren más expuestos a padecerlas. Disponemos de gran cantidad de indicios sobre los cuales iniciar acciones eficaces para proteger la salud cardiovascular, especialmente mediante medidas orientadas a la población para prevenir los principales factores de riesgo; para lograrlo es necesario que previamente se identifiquen los determinantes de esta enfermedad y las poblaciones más susceptibles de padecerlas, de manera que sea posible aplicar en ellos medidas de prevención y promoción de la salud. ${ }^{1}$

Los trabajos con largas jornadas laborales, sedentarismo y que no suelen permitir desarrollar un estilo de vida saludable podrían influir en el desarrollo de una patología cardiovascular. En base a esto, ciertas profesiones podrían asociarse con un mayor riesgo de padecer enfermedad cardiovascular, entre ellas podríamos citar a los conductores. Algunos autores afirman que existen ciertos factores de riesgo modificables como el alcohol, el tabaco y la alimentación rica en lípidos y sal, que afectan la salud de los conductores. ${ }^{2}$ A los trabaja- 
dores que conducen una gran cantidad de horas, les puede resultar muy complicado llevar a cabo una alimentación sana, realizar actividad física habitual y controlar su peso. Creemos, pues, que la profesión de conductor se podría considerar como de alto riesgo para padecer ECV; por ello realizamos este trabajo que tiene como objetivo determinar el nivel de riesgo cardiovascular que tienen diferentes trabajadores del sector de la conducción, valorando igualmente qué factores influyen en la aparición o no de estas patologías.

\section{MATERIAL Y MÉTODOS}

\section{Características del estudio}

Se realiza un estudio retrospectivo y transversal en 2944 varones conductores profesionales (487 taxistas, 908 conductores de autobús y 1549 conductores de camión) del área mediterránea española en el periodo comprendido entre enero de 2015 y diciembre de 2016. Los trabajadores fueron seleccionados entre los que acudieron a los reconocimientos médicos laborales periódicos. Se informó a los Comités de Seguridad y Salud de las diferentes empresas y se pidió el consentimiento informado a todas las personas que entraron en el estudio para cumplir con la legislación vigente. El protocolo del estudio estaba de acuerdo con la Declaración de Helsinki. Después de la aceptación, se registró un historial médico completo, incluyendo historia familiar e historia personal.

\section{Criterios de inclusión/exclusión}

Como criterios de inclusión se consideran: ser varón (no se incluyeron las mujeres por ser un número mínimo), conductor profesional, aceptar el uso de los datos obtenidos en los reconocimientos médicos con fines epidemiológicos, pertenecer a alguna de las empresas incluidas en el estudio. Como criterios de exclusión se considera: la no aceptación del trabajador en el uso de los datos para el estudio, no realizarse extracción de sangre por laboratorio concertado y no pertenecer a alguna de las empresas participantes. En el periodo citado, 3104 conductores se realizaron reconocimientos médicos laborales, fueron descartados 98 trabajadores, 38 mujeres, 12 por no ceder el uso de sus datos, 45 por no realizarse extracción para muestra sanguínea y 3 por no estar en el tramo de edad considerado en el estudio.

\section{Determinación de variables}

Las diferentes mediciones antropométricas, clínicas y las extracciones para las analíticas fueron realizadas por el personal de Enfermería del Trabajo de los diferentes servicios de prevención que intervenían en el estudio.

Las medidas antropométricas se realizaron siguiendo las normas de los International Standars for Anthropometric Assessment ${ }^{3}$, determinándolas por triplicado y utilizando la media. El peso, con ajuste a $0,1 \mathrm{~kg}$, y la talla con ajuste a 0,5 cm, se determinaron, respectivamente, utilizando báscula y estadiómetro estandarizados (Seca 700 y Seca 220). Se calculó el índice de masa corporal (IMC) y se definió sobrepeso y obesidad según los criterios de la Organización mundial de la Salud (OMS): ${ }^{4}$ obesidad si IMC $\geq 30 \mathrm{~kg} / \mathrm{m} 2$ y sobrepeso si IMC $\geq 25 \mathrm{~kg} / \mathrm{m} 2 \mathrm{y}<30 \mathrm{~kg} / \mathrm{m} 2$. El perímetro de cintura abdominal se calculó con una cinta métrica colocada paralela al suelo a nivel de la última costilla flotante, es decir, se mide el contorno del talle natural tomado entre la parte superior del hueso de la cadera (crestas ilíacas) y la costilla inferior, medido durante la respiración normal con el sujeto de pie y con el abdomen relajado. El colesterol total (CT) y los triglicéridos se determinaron por
$\mathrm{Cl} 2 \mathrm{Mg}$, el colesterol ligado a lipoproteínas de alta densidad (C-HDL) se calculó métodos enzimáticos automatizados, el C-LDL se determinó por precipitación con dextrano-sulfato mediante la fórmula de Friedwald (siempre que los triglicéridos fueron $<400$ $\mathrm{mg} / \mathrm{dl})$. La extracción de sangre se realizó en la misma sesión y en el mismo lugar, tras ayuno nocturno de 12 horas. Las muestras se remitieron al laboratorio de referencia y se procesaron en un máximo de 72 horas, conservándolas a una temperatura de $-20^{\circ} \mathrm{C}$. Se consideraron valores limitrofes cuando el colesterol estaba comprendido entre 200 y $239 \mathrm{mg} / \mathrm{dl}$, el LDL entre 130-159 mg/dl y los triglicéridos entre 150 y $199 \mathrm{mg} / \mathrm{dl}$ y valores elevados cuando el colesterol era igual o superior a $240 \mathrm{mg} / \mathrm{dl}$, el LDL era igual o superior a $160 \mathrm{mg} / \mathrm{dl}$ y los triglicéridos iguales o superiores a 200 $\mathrm{mg} / \mathrm{dl}$. La glucosa se determinó por un método enzimático y se consideró prediabetes a valores entre 100 y $125 \mathrm{mg} / \mathrm{dl}$ e hiperglucemia cuando los valores eran iguales o superiores a $126 \mathrm{mg} / \mathrm{dl}$.

La presión arterial se determinó empleando un esfigmomanómetro automático OMRON M3 calibrado; tras un periodo de reposo de 10 minutos en decúbito supino, con tres mediciones realizadas a intervalos de 1 minuto de separación entre ellas, calculándose la media de las tres mediciones. Se consideró HTA cuando los valores eran iguales o superiores a 140/90 $\mathrm{mmHg}$.

Para calcular los diferentes indices aterogénicos se emplearon las siguientes fórmulas:

- índice aterogénico de Castelli ${ }^{5}=$ Colesterol total/c-HDL.

- índice aterogénico de Kannel = c-LDL/c-HDL.

- índice aterogénico triglicéridos/c-HDL.

Para cada indice se establecieron diferentes puntos de corte de acuerdo a los datos existentes en la bibliografía:

Se consideró índice de Castelli con riesgo bajo ${ }^{6}$ si los valores eran inferiores a 4,5\% en mujeres e inferiores a 5\% en hombres, moderado entre 4,5-7\% en mujeres y 5-9\% en hombres y alto si era superior al 7 y 9\% respectivamente. Índice de Kannel de riesgo bajo si era inferior a 3\% y alto a partir de ese valor. El índice triglicéridos/c-HDL se considera elevado a partir de 3\%.

REGICOR es el modelo de Framingham calibrado para población española ${ }^{7,8}$ que emplea como parámetros el sexo, la edad, el consumo de tabaco, la tensión arterial sistólica y diastólica, el colesterol total, HDL y la diabetes. Los cálculos se pueden hacer en personas entre 35 y 74 años. La catalogación de REGICOR es: bajo (< 5\%), moderado (entre 5 y 9.9\%), alto (entre 10 y 14.9\%) y muy alto (a partir de 15\%). El modelo DORICA ${ }^{9}$ se construyó a partir de datos de poblaciones de diferentes comunidades autónomas españolas e incluye población entre 25 y 64 años. Los parámetros que utiliza son los mismos del REGICOR. La catalogación es: bajo $(<5 \%$ ), ligeramente elevado (entre 5 y 9\%), moderado (entre 10 y 19\%), alto (entre 20 y 39\%) y muy alto (a partir de 40\%).

El modelo SCORE predice la mortalidad cardiovascular en 10 años y hay tablas específicas para población española entre 40 y 65 años. ${ }^{10}$ Los puntos de corte recomendados son: bajo si es inferior al 3\%, moderado entre 3\% y 4,9\% y alto desde 5\%. Las variables utilizadas son: edad, sexo, consumo de tabaco, presión arterial sistólica, colesterol, colesterol HDL.

La edad del corazón ${ }^{11,12}$ se calculó mediante la herramienta denominada "Heart age calculator", disponible en la página web: www. heartage.me. El peso de cada uno de los parámetros analizados sobre la edad del corazón es similar al que tienen esos mismos factores en la determinación del riesgo cardiovascular según el modelo de Framingham. Los parámetros necesarios para el cálculo de la edad 
del corazón son los siguientes: edad, sexo, altura (en centímetros), peso (en kilogramos), perímetro de cintura (en centímetros), antecedentes de enfermedad cardiovascular de madre o padre y edad que tenían cuando la sufrieron por primera vez, presencia o no de diabetes, consumo de tabaco (si no se fuma en la actualidad también se pregunta si se ha abandonado el consumo en el último año), valores de colesterol total y HDL colesterol y finalmente valores de presión sistólica y si se está actualmente en tratamiento antihipertensivo. Al restar del valor obtenido de la herramienta la edad real obtenemos el número de años que la persona ha "ganado o perdido". La edad vascular es una herramienta basada en la escala SCORE. ${ }^{13} \mathrm{La}$ edad vascular de una persona es igual a la edad que tendría una persona con el mismo nivel de riesgo cardiovascular estimado, pero con todos los factores de riesgo en niveles normales. En el año $2010^{13}$ se publicaron las tablas para poder calcularla.

Para determinar la existencia de síndrome metabólico se emplearon dos modelos: NCEP ATP III (National Cholesterol Educational Program Adult Treatment Panel III) que establecen síndrome metabólico cuando tres o más de los siguientes factores están presentes: perímetro de cintura mayor de $88 \mathrm{~cm}$ en mujeres y 102 en hombres, triglicéridos a partir de $150 \mathrm{mg} / \mathrm{dl}$ o tratamiento

específico de esta alteración lipídica, presión arterial a partir de 130/85 mm Hg, HDL inferior a $40 \mathrm{mg} / \mathrm{dl}$ en mujeres o inferior a 50 en hombres o tratamiento específico, glucemia en ayunas a partir de $100 \mathrm{mg} / \mathrm{dl}$ o tratamiento específico de glucemia y el modelo de la International Diabetes Federation (IDF) que indican como factor necesario la presencia de obesidad central, definida como un perímetro de cintura a partir de $80 \mathrm{~cm}$ en mujeres y $94 \mathrm{~cm}$ en hombres, más al menos dos de los otros factores antes señalados para ATP III (triglicéridos, HDL, tensión arterial y glucemia).

El cuestionario FINDRISC valora 8 items: edad, IMC, perímetro de cintura, actividad física, alimentación, uso de medicación antihipertensiva, antecedentes personales de cifras elevadas de glucemia e historia familiar de diabetes. La puntuación del test va de 0 a 26 puntos, a partir de los cuales se establece el nivel de riesgo de padecer diabetes tipo 2 en los próximos 10 años: de 0 a 7 puntos, bajo riesgo; de 7 a 11 puntos, riesgo ligeramente elevado; de 11 a 14 puntos, riesgo moderado; de 15 a 20 puntos, riesgo alto, y más de 20 puntos, riesgo muy alto.

El QDScore es el primer algoritmo de predicción de riesgo para estimar el riesgo de diabetes a 10 años. El algoritmo no necesita pruebas de laboratorio y puede ser utilizado en entornos clínicos y también por el público general a través de una simple calculadora web (www.qdscore.org).

El tipo de conductor se determinó a partir de la clasificación nacional de ocupaciones del año $2011^{14}$ (CNO-2011) y se clasificaron en tres categorías: conductores de taxi (código 841), de autobús (código 842) y de camión (código 843).

Se considera consumo de alcohol cuando se superan las tres unidades de bebida estándar (UBE) al día en hombres, teniendo en cuenta que una UBE equivale a 10 gramos de alcohol. Se considera actividad física cuando se realiza habitualmente al menos 30 minutos al día o 4 horas semanales tanto en el trabajo como en el tiempo libre. La alimentación se considera adecuada cuando se consumen frutas y verduras todos los días. Se considera fumador a aquella persona que ha fumado diariamente durante el último mes cualquier cantidad de cigarrillos.

\section{Análisis estadístico}

Cuando la variable es continua se comparan las medias mediante la prueba $\mathrm{t}$ de Student-Fischer si la variable sigue una distribución normal, o con la prueba no paramétrica test de U de Mann-Whitney
Tabla 1.

Características de la muestra.

$\begin{array}{lccccc} & \begin{array}{c}\text { autobús } \\ n=908 \\ \text { media }(\mathrm{dt})\end{array} & \begin{array}{c}\text { camión } \\ \mathrm{n}=1549 \\ \text { media }(\mathrm{dt})\end{array} & \begin{array}{c}\text { taxi } \\ \mathrm{m}=487 \\ \text { media }(\mathrm{dt})\end{array} & \begin{array}{c}\text { total } \\ \mathrm{n}=2944 \\ \text { media (dt) }\end{array} & \mathrm{p} \\ \text { edad } & 44,3(10,0) & 40,7(9,6) & 40,6(10,7) & 41,8(10,1) & <0,0001 \\ \text { altura } & 172,8(6,9) & 173,9(6,6) & 173,5(6,8) & 173,5(6,7) & <0,0001 \\ \text { peso } & 84,1(12,8) & 84,5(14,3) & 83,4(14,7) & 84,2(13,9) & 0,279 \\ \text { p cintura } & 91,2(10,2) & 89,6(9,4) & 90,5(11,0) & 90,2(10,0) & 0,001 \\ \text { TAS } & 124,8(15,3) & 126,8(15,3) & 126,5(15,7) & 126,1(15,4) & 0,010 \\ \text { TAD } & 75,5(10,5) & 76,6(10,6) & 76,3(11,3) & 76,2(10,7) & 0,032 \\ \text { colesterol } & 203,0(38,7) & 197,7(39,1) & 200,0(40,3) & 199,7(39,2) & 0,006 \\ \text { HDL-c } & 48,1(7,6) & 50,2(6,8) & 49,8(8,7) & 49,5(7,4) & <0,0001 \\ \text { LDL-c } & 127,1(38,7) & 121,7(38,9) & 122,0(37,7) & 123,4(38,7) & 0,003 \\ \text { triglicéridos } & 141,7(102,0) & 131,9(101,1) & 144,4(109,3) & 137,0(102,9) & 0,016 \\ \text { glucemia } & 96,5(24,1) & 91,5(21,9) & 99,0(30,1) & 94,3(24,3) & <0,0001 \\ & \% & \% & \% & \% & \\ \text { tabaco } & 34,9 & 38,9 & 34,9 & 37,0 & 0,079 \\ \text { ejercicio físico } & 30,9 & 37,7 & 34,3 & 35,1 & 0,003 \\ \text { alimentación } & 29,3 & 34,7 & 31,2 & 32,4 & 0,019 \\ \text { alcohol } & 26,3 & 17,4 & 15,0 & 19,8 & <0,0001\end{array}$

si no se cumple el principio de normalidad. Si la variable es cualitativa se comparan las proporciones mediante la prueba chi cuadrado de Pearson. El análisis multivariante se realizó mediante regresión logística multinomial. En todos los análisis se aceptó como nivel de significación un valor de $\mathrm{p}$ inferior a 0,05.

\section{RESULTADOS}

El estudio se ha realizado en 2944 varones conductores profesionales; las características de la muestra en cuanto a los valores de los diferentes parámetros relacionados con el riesgo cardiovascular se muestran en la Tabla 1.

Se determina el valor medio de diferentes indicadores de riesgo cardiovascular en cada uno de los colectivos estudiados y se observa que los mejores valores los obtienen los conductores de camión en casi todos los casos. Los datos completos se muestran en la Tabla 2.

\section{Tabla 2.}

Valores medios de diferentes indicadores de riesgo cardiovascular según tipo de conductor.

\begin{tabular}{|c|c|c|c|c|c|}
\hline & $\begin{array}{c}\text { autobús } \\
n=908 \\
\text { media (dt) }\end{array}$ & $\begin{array}{c}\text { camión } \\
n=1549 \\
\text { media (dt) }\end{array}$ & $\begin{array}{c}\text { taxi } \\
n=487 \\
\text { media (dt) }\end{array}$ & $\begin{array}{c}\text { total } \\
\mathrm{n}=2944 \\
\text { media (dt) }\end{array}$ & $p$ \\
\hline IMC & $28,2(4,0)$ & $27,9(4,4)$ & $27,7(4,4)$ & $28,0(4,3)$ & 0,094 \\
\hline cintura/altura & $0,53(0,06)$ & $0,52(0,05)$ & $0,52(0,06)$ & $0,52(0,06)$ & ) $<0,0001$ \\
\hline colesterol/HDL-c & & & $4,2(1,4)$ & $4,2(1,3)$ & $<0,0001$ \\
\hline LDL-c/HDL-c & 2,8 & & 2,6 & ,1) & $<0,0001$ \\
\hline triglicéridos/HDL-c & & & & & 0,001 \\
\hline HDL-c/(LDL-c+VLDL-c) & $0,3($ & 0,4 & 0,4 & $0,4(0,2)$ & $<0,0001$ \\
\hline sd metabólico ATPIII & 1,4 & 1 & 1,4 & ,2) & $<0,0001$ \\
\hline REGICOR & 3,7 & 3,4 & 3,9 & 3,6 & 0,004 \\
\hline DOR & 6,0 & 5 & 6,3 & $1,9)$ & $<0,0001$ \\
\hline SCORE & $1,6(2$ & $1,3(1,5)$ & $1,6(1,7)$ & $1,5(1,8)$ & 0,002 \\
\hline años perdidos edad corazón & n $7,3(8,1)$ & $7,3(7,9)$ & $7,2(8,7)$ & $7,3(8,1)$ & 0,966 \\
\hline años perdidos edad vascular & $4,1(6,0)$ & $4,6(6,0)$ & $5,5(5,9)$ & $4,6(6,0)$ & 0,009 \\
\hline riesgo relativo Qdscore & $1,6(1,8)$ & $1,5(1,8)$ & $1,6(1,9)$ & $1,6(1,8)$ & 0,466 \\
\hline Findrisk & $6,9(5,0)$ & $5,6(4,5)$ & $5,7(4,7)$ & $6,0(4,7)$ & $<0,0001$ \\
\hline
\end{tabular}


En lo que hace referencia a la prevalencia de valores alterados de las diferentes escalas analizadas, se observa que en casi todos los casos las prevalencias más bajas aparecen en el colectivo de camioneros mientras que las más altas son más frecuentes entre los conductores de autobús. Los datos completos se presentan en la Tabla 3.

Tabla 3.

Prevalencia de valores alterados en las diferentes escalas de riesgo cardiovascular según tipo de conductor.

\begin{tabular}{|c|c|c|c|c|c|c|c|c|c|}
\hline & $\begin{array}{c}\text { autobú } \\
n\end{array}$ & & $\underset{n}{\text { camión }}$ & & $\begin{array}{c}\operatorname{taxi} \\
\mathrm{n}\end{array}$ & & $\underset{n}{\text { total }}$ & & $p$ \\
\hline sobrepeso & 908 & 49,1 & 1549 & 47,8 & 487 & 44,4 & 2944 & 47,6 & 0,099 \\
\hline obesidad & & 28,9 & & 26,9 & & 26,3 & & 27,4 & \\
\hline cintura/altura $>0,5$ & 908 & 66,3 & 1549 & 59,1 & 487 & 60,0 & 2944 & 61,5 & 0,002 \\
\hline pre HTA & 908 & 52,1 & 1549 & 52,9 & 487 & 53,8 & 2944 & 52,8 & 0,001 \\
\hline HTA 1 & & 17,1 & & 20,5 & & 17,5 & & 18,9 & \\
\hline HTA 2 & & 4,6 & & 6,1 & & 8,6 & & 6,1 & \\
\hline colesterol $200-239 \mathrm{mg} / \mathrm{dl}$ & 908 & 33,3 & 1549 & 34,2 & 487 & 32,6 & 2944 & 33,7 & 0,082 \\
\hline colesterol $\geq 240 \mathrm{mg} / \mathrm{dl}$ & & 16,6 & & 12,6 & & 15,2 & & 14,3 & \\
\hline $\mathrm{HDL}-\mathrm{c}<40 \mathrm{mg} / \mathrm{dl}$ & 908 & 15,2 & 1549 & 7,1 & 487 & 16,0 & 2944 & 11,1 & $<0,0001$ \\
\hline LDL-c 130-159 mg/dl & 908 & 25,7 & 1549 & 27,7 & 487 & 23,4 & 2944 & 26,4 & 0,012 \\
\hline $\mathrm{LDL}-\mathrm{c} \geq 160 \mathrm{mg} / \mathrm{dl}$ & & 18,3 & & 13,5 & & 16,2 & & 15,4 & \\
\hline triglicéridos 150-199 mg/dl & 908 & 16,6 & 1549 & 12,6 & 487 & 12,7 & 2944 & 13,9 & 0,002 \\
\hline triglicéridos $\geq 200 \mathrm{mg} / \mathrm{dl}$ & & 16,1 & & 14,1 & & 19,3 & & 15,6 & \\
\hline glucemia $100-125 \mathrm{mg} / \mathrm{dl}$ & 908 & 23,1 & 1549 & 15,5 & 487 & 18,5 & 2944 & 18,3 & $<0,0001$ \\
\hline glucemia $\geq 126 \mathrm{mg} / \mathrm{dl}$ & & 6,7 & & 4,3 & & 13,1 & & 6,5 & \\
\hline col/HDL-c moderado & 908 & 25,6 & 1549 & 15,8 & 487 & 21,4 & 2944 & 19,7 & $<0,0001$ \\
\hline $\mathrm{col} / \mathrm{HDL}-\mathrm{c}$ alto & & 0,8 & & 0,3 & & 0,6 & & 0,5 & \\
\hline LDL-c/HDL-c alto & 908 & 22,6 & 1549 & 13,2 & 487 & 17,2 & 2944 & 16,8 & $<0,0001$ \\
\hline triglicéridos/ HDL-c > 3 & 908 & 34,9 & 1549 & 28,1 & 487 & 33,1 & 2944 & 31,0 & 0,001 \\
\hline sd metabólico ATP III & 908 & 18,7 & 1549 & 12,3 & 487 & 21,1 & 2944 & 15,8 & $<0,0001$ \\
\hline sd metabólico IDF & 908 & 18,1 & 1549 & 13,4 & 487 & 19,5 & 2944 & 15,8 & \\
\hline REGICOR moderado & 743 & 3,0 & 1095 & 1,9 & 337 & 3,6 & 2175 & 2,5 & $<0,0001$ \\
\hline REGICOR alto & & 0,0 & & 0,3 & & 0,0 & & 0,1 & \\
\hline DORICA moderado & 887 & 18,7 & 1496 & 12,2 & 448 & 17,0 & 2831 & 15,0 & $<0,0001$ \\
\hline DORICA alto-muy alto & & 2,8 & & 2,5 & & 4,5 & & 2,9 & \\
\hline SCORE medio & 605 & 6,0 & 813 & 3,2 & 243 & 4,9 & 1661 & 4,5 & $<0,0001$ \\
\hline SCORE alto & & 4,0 & & 2,8 & & 4,1 & & 3,4 & \\
\hline Finrisk moderado & 856 & 11,9 & 1497 & 6,9 & 426 & 6,8 & 2779 & 8,5 & $<0,0001$ \\
\hline Finrisk alto-muy alto & & 6,8 & & 3,3 & & 4,2 & & 4,5 & \\
\hline
\end{tabular}

En el análisis multivariante se establecen como categorias de referencia el ser conductor de taxi, edad a partir de 60 años, ser fumador, no hacer ejercicio físico y no tener una alimentación cardiosaludable. En las variables antropométricas, clínicas y analíticas el tipo de conductor solo se relaciona con la aparición de hipertensión, hipertrigliceridemia e hiperglucemia, observándose que los taxistas presentan una odss ratio de 1,7 (IC 95\% 1,3-2,2) frente a los conductores de autobús en la aparición de hipertensión, 1,5 (IC 95\% 1,1-2) frente a los camioneros y 1,4 (IC 95\% 1-1,99) frente a los conductores de autobús en la aparición de hipertrigliceridemia y 3,7 (IC 95\% 2,5-5,4) frente a camioneros y 3,4 (IC 95\% 2,2-5) frente a conductores de autobús en hiperglucemia; los datos completos se muestran en la Tabla 4a. Los taxistas presentan mayor riesgo de presentar índices aterogénicos elevados que los camioneros (salvo triglicéridos/HDL mayor de 3) y también síndrome metabólico, frente a los conductores de autobús solo presentan mayor riesgo de sufrir síndrome metabólico con el modelo ATP III; podemos ver los datos completos en la Tabla 4b. En cuanto a las escalas de riesgo cardiovascular y riesgo de diabetes tipo 2, solo se observa una relación con el tipo de conductor en la escala DORICA, siendo las odss ratio de 1,7 de los taxistas frente a los camioneros (IC 95\% 1,2-2,4) y de 1,6 (IC 95\% 1,1-2,3) para los conductores de autobús; los datos completos se encuentran en la Tabla 4c.

\section{DISCUSIÓN}

El nivel de riesgo cardiovascular de los conductores profesionales se puede considerar globalmente como elevado, ya que la mayoría de las variables analizadas presentan prevalencia de valores alterados muy destacables.

No hemos encontrado en la literatura científica consultada un estudio como el nuestro que valore en el mismo trabajo diferentes tipos de conductores y sus niveles de riesgo cardiovascular; por lo tanto, no será posible comparar nuestros resultados con los obtenidos por otros autores siguiendo esta metodología. Por ello deberemos comparar nuestros resultados con los obtenidos por otros investigadores haciendo referencia a cada tipo de conductor por separado. La mayoría de estudios consultados presentan tamaños muestrales muy bajos, generalmente inferiores a 100 conductores, por lo que las prevalencias obtenidas en ellos al ser comparadas con nuestro trabajo realizado en casi 3000 conductores podrán diferir.

En nuestro trabajo, alrededor del 75\% de los conductores presentan sobrepeso u obesidad; estos datos son similares a los obtenidos en estudios realizados en poblaciones de la India $^{15}$ y Brasil ${ }^{16}$, y algo superiores a los obtenidos en población coreana. ${ }^{17}$ La prevalencia de obesidad en nuestros conductores estaba entre el 25-30\% algo inferior a la encontrada en conductores profesionales de Hong Kong ${ }^{18}$ que era superior al 50\%. La prevalencia de hipertensión en nuestro trabajo era elevada, alrededor del 25\%, pero inferior a la encontrada por otros autores en población colombiana ${ }^{19}$, hindú ${ }^{15}$ y brasileña. ${ }^{20}$ El perfil lipídico alterado en nuestro colectivo es muy alto, tanto en lo referente a colesterol total como a HDL, LDL y triglicéridos, y es superior al encontrado en el estudio colombiano ${ }^{19}$ y claramente inferior al realizado en conductores de Hong Kong. ${ }^{18}$ La prediabetes afecta al 18,3\% de nuestros conductores y la diabetes al 6,5\%; estas cifras son superiores a las obtenidas por Sangaleti ${ }^{16}$ e inferiores a las que presentó en su estudio Hirata et al. ${ }^{20}$

Nuestros conductores presentan una prevalencia de síndrome metabólico con los modelos ATPIII e IDF del 15,8\% que es netamente superior al de los trabajadores de Hong Kong ${ }^{18}$ y claramente inferior al de los trabajadores coreanos. ${ }^{17}$

El riesgo cardiovascular con el modelo SCORE en nuestros conductores fue considerado medio en el 6\% y alto en un 4\%; estas prevalencias son superiores a las encontradas en conductores de autobús colombianos. ${ }^{19}$

No hemos encontrado trabajos como el nuestro que valoren el riesgo cardiovascular de conductores empleando las escalas REGICOR, DORICA, edad del corazón o edad vascular, ni tampoco estudios donde se analice el riesgo de sufrir diabetes tipo 2 con los modelos Findrisk o QDscore.

$\mathrm{Al}$ investigar el estilo de vida de nuestros conductores, vemos que un $37 \%$ de los trabajadores de nuestra muestra son fumadores activos, el 35,1\% realizan ejercicio físico regular al menos tres días a la semana, un 32,4\% tiene una alimentación cardiosaludable rica en frutas y verduras y un 19,8\% consumen más de 3 UBE al día. El consumo de tabaco es superior al obtenido en el trabajo de Sangaleti et $\mathrm{al}^{16} \mathrm{y}$ en otro realizado en población colombiana. ${ }^{19} \mathrm{El}$ nivel de actividad física es inferior al encontrado en otros trabajos ${ }^{20-21} \mathrm{y}$ similar al encontrado en población estadounidense. ${ }^{22}$ El consumo de alcohol de nuestros conductores, pese a ser elevado (19,8\%), es claramente inferior al del estudio de Sangaleti ${ }^{16}$ (aunque en este caso se valora el consumo o no de alcohol de forma regular sin cuantificar si es o no muy elevado como hemos hecho nosotros), y otros realizados en Andra Pradesh ${ }^{15}$ y en población colombiana. ${ }^{19}$ 
Tabla 4a.

Análisis multivariante para variables antropométricas, clínicas y analíticas relacionadas con riesgo cardiovascular.

\begin{tabular}{|c|c|c|c|c|c|c|c|c|c|c|c|c|}
\hline & $\begin{array}{c}\text { obesidad } \\
\text { OR (IC 95\%) }\end{array}$ & $p$ & $\begin{array}{c}\text { HTA } \\
\text { OR (IC 95\%) }\end{array}$ & $p$ & $\begin{array}{l}\text { colesterc } \\
\text { OR (IC }\end{array}$ & $p$ & $\begin{array}{l}\text { LDL } \\
\text { OR (IC }\end{array}$ & $p$ & $\begin{array}{c}\text { triglicéridos } \geq 200 \\
\text { OR (IC } 95 \% \text { ) }\end{array}$ & $p$ & $\begin{array}{l}\geq 126 \\
5 \%)\end{array}$ & 6 \\
\hline utobús & 0,9 & 699 & $1,7(1,3-2,2)$ & 0.0001 & 1 & 360 & & 0.962 & & 0.030 & $-5,0)$ & $<0.0001$ \\
\hline $0-39 a$ & $1,3($ & 0.218 & $7,3(4,9-10,9)$ & $<0.0001$ & 8) & 0.841 & 1,4 & 0.220 & 0,6 & 0.115 & 37,6 & ) $<0.0001$ \\
\hline $40-49 a$ & $1,3(0,8-1,9)$ & 0.272 & $4,0(2,7-5,9)$ & $<0.0001$ & $0,7(0$ & 0.168 & $0,9($ & 0.559 & 0,6 & 0.077 & $-12,9)$ & $<0.0001$ \\
\hline $50-59 a$ & $0,9(0$ & 0.533 & $2,2(1,5-3,2)$ & $<0.0001$ & $0,7($ & 0.218 & 0,8 & 0.380 & & 0.110 & $7-4,3)$ & $<0.0001$ \\
\hline i alimentaci & $86,3(47,2-116,9)$ & $<0.0001$ & $0,8(0,5-1,3)$ & 0.463 & $13,5(1,7-111,2)$ & 0.015 & $12,2(4,8-31,2)$ & $<0.0001$ & $2,0(0,8-5,2)$ & 0.150 & $1,0(0,4-2,5)$ & 0.979 \\
\hline
\end{tabular}

Tabla 4b.

Análisis multivariante para índices aterogénicos y síndrome metabólico.

\begin{tabular}{|c|c|c|c|c|c|c|c|c|c|c|}
\hline & $\begin{array}{c}\text { CT/HDL elevado } \\
\text { OR (IC 95\%) }\end{array}$ & $\mathrm{p}$ & $\begin{array}{l}\text { LDL/HDL alto } \\
\text { OR (IC 95\%) }\end{array}$ & $\mathrm{p}$ & $\begin{array}{l}\mathrm{TG} / \mathrm{HDL}>3 \\
\text { OR (IC 95\%) }\end{array}$ & $\mathrm{p}$ & $\begin{array}{l}\text { sd mtb ATP III } \\
\text { OR (IC 95\%) }\end{array}$ & $\mathrm{p}$ & $\begin{array}{l}\text { sd mtb IDF } \\
\text { OR (IC 95\%) }\end{array}$ & $\mathrm{p}$ \\
\hline camión & $1,5(1,1-2,0)$ & 0.004 & $1,4(1,0-1,8)$ & 0.038 & $1,3(1,0-1,7)$ & 0.056 & $2,0(1,5-2,7)$ & $<0.0001$ & $1,6(1,2-2,1)$ & 0.002 \\
\hline autobús & $0,9(0,7-1,3)$ & 0.682 & $0,8(0,6-1,1)$ & 0.265 & $1,1(0,8-1,4)$ & 0.721 & $1,5(1,1-2,0)$ & 0.014 & $1,3(1,0-1,8)$ & 0.069 \\
\hline $30-39 a$ & $2,9(1,8-4,5)$ & $<0.0001$ & $2,3(1,4-3,7)$ & 0.001 & $1,2(0,8-1,9)$ & 0.360 & $2,9(1,8-4,7)$ & $<0.0001$ & $1,6(1,0-2,7)$ & 0.063 \\
\hline $40-49 a$ & $1,3(0,8-1,9)$ & 0.290 & $1,2(0,8-1,8)$ & 0.479 & $1,0(0,6-1,5)$ & 0.855 & $1,6(1,0-2,6)$ & 0.036 & $0,9(0,5-1,4)$ & 0.518 \\
\hline $50-59 a$ & $0,9(0,6-1,5)$ & 0.789 & $0,8(0,5-1,3)$ & 0.364 & $0,9(0,6-1,3)$ & 0.467 & $0,9(0,6-1,4)$ & 0.582 & $0,6(0,4-1,0)$ & 0.042 \\
\hline sí alimentación & $0,9(0,4-1,8)$ & 0.750 & $0,7(0,3-1,4)$ & 0.300 & $1,5(0,8-2,8)$ & 0.175 & $3,5(1,2-10,1)$ & 0.021 & $2,1(0,8-5,4)$ & $0.120 \mathrm{E}$ \\
\hline
\end{tabular}

CT/HDL elevado: moderado o alto.

Tabla 4c.

Análisis multivariante para escalas de riesgo cardiovascular y de riesgo de diabetes tipo 2.

\begin{tabular}{|c|c|c|c|c|c|c|c|c|}
\hline & REGICOR elevado & & DORICA elevado & & SCORE elevado & & Findrisk elevado & \\
\hline autobús & $1,7(0,8-3,7)$ & 0.194 & $1,6(1,1-2,3)$ & 0.012 & $1,1(0,6-1,9)$ & 0.814 & $0,7(0,5-1,0)$ & 0.079 \\
\hline $30-39 a$ & $89,1(21,3-110,8)$ & $<0.0001$ & $554,5(254,9-1206,0)$ & $<0.0001$ & & & $7,0(3,9-12,3)$ & $<0.0001$ \\
\hline $40-49 a$ & $36,4(12,4-106,9)$ & $<0.0001$ & $39,8(23,7-66,9)$ & $<0.0001$ & $124,3(63,7-242,7)$ & $<0.0001$ & $2,7(1,6-4,4)$ & $<0.0001$ \\
\hline $50-59 a$ & $2,3(1,2-4,5)$ & 0.018 & $5,6(3,4-9,1)$ & $<0.0001$ & $8,0(3,2-12,9)$ & $<0.0001$ & $1,1(0,6-1,8)$ & 0.781 \\
\hline si alimentación & $84,3(18,2-186,7)$ & $<0.0001$ & $1,7(0,8-3,7)$ & 0.198 & $1,4(0,5-4,0)$ & 0.523 & $7,3(1,4-38,0)$ & 0.018 \\
\hline
\end{tabular}

REGICOR elevado: moderado o alto. DORICA elevado: moderado, alto y muy alto. SCORE elevado: medio y alto. Findrisk elevado: moderado, alto y muy alto. 


\section{REFERENCIAS}

1. Somoza MI, Torresani ME. Lineamientos para el cuidado nutricional. Buenos Aires: Eudeba; 2007.

2. Mota Guedes H, de Arêdes Brum K, Andrade Costa P, Ferreira de Almeida ME. Fatores de risco para o desenvolvimento de hipertensao arterial entre motoristas caminhoneiros. Cogitare Enferm. 2010; 15(4):652-658.

3. ISAK. International standars for anthropometric assessment. Underdale: ISAK; 2001.

4. WHO. Obesity: preventing and managing the global epidemic; Report of a WHO consultation. Geneva: WHO; 2000.

5. Castelli WP. Epidemiology of coronary heart disease; The Framingham Study. Am J Med. 1984; 76:4-9.

6. Orgaz-Morales MT, Hijano-Villegas S, Martínez-Llamas MS, López-Barba J, Díaz-Portillo J. Guía del paciente con trastornos lipídicos. Ministerio de Sanidad y Consumo. Madrid: Instituto Nacional de Gestión Sanitaria; 2007.

7. Marrugat J, Subirana I, Comin E, Cabezas C, Vila J, Elosua R, et al. Validity of an adaptation of the Framingham cardiovascular risk function; the VERIFICA Study. J Epidemiol Comm Health. 2007; 61(1):40-47.

8. Marrugat J, Vila J, Baena-Diez JM, Grau M, Sala J, Ramos R et al. Validez relativa de la estimación del riesgo cardiovascular a 10 años en una cohorte poblacional del estudio REGICOR. Rev Esp Cardiol. 2011; 64(5):385-394.

9. Aranceta J, Pérez-Rodrigo C, Foz-Sala M, Mantilla T, Serra-Majem L, Moreno B et al. (Grupo colaborativo para el estudio DORICA fase II). Tablas de evaluación del riesgo coronario adaptadas a la población española; Estudio DORICA. Med Clin (Barc). 2004; 123(18):686-691.

10. Sans $S$, Fitzgerald AP, Royo D, Conroy D, Graham I. Calibración de la tabla SCORE de riesgo cardiovascular para España. Rev Esp Cardiol. 2007; 60:476485.

11. D'Agostino RB, Vasan RS, Pencina MJ, Wolf PA, Cobain M, Massaro JM, Kannel WB. General cardiovascular risk profile for use in primary care; The Framingham Heart Study. Circulation. 2008; 117:743-753.

12. López-González AA, Aguilo A, Frontera M, Bennasar-Veny M, Campos I, Vicente-Herrero T, Tomás-Salva M, De Pedro-Gómez J, Tauler P. Effectiveness of the Heart Age tool for improving modifiable cardiovascular risk factors in a Southern European population: a randomized trial. Eur J Prev Cardiol. 2015; 22:389-396.

13. Cuende Jl, Cuende N, Calaveras-Lagartos J. How to calculate vascular age with the SCORE Project scales: a new method of cardiovascular risk evaluation. Eur Heart J. 2010; 31(9):2351-2358.

14. Domingo-Salvany A, Bacigalupe A, Carrasco JM, Espelt A, Ferrando J, Borrel C. Propuesta de clase social neoweberiana y neomarxista a partir de la Clasificación Nacional de Ocupaciones 2011. Gac Sanit. 2013; 27(3):263272.

15. Eshwaran Udayar S, Kumar KR, Kumar P, Vairamuthu S, Thatuku S. Study of cardiovascular risk factors among Transport drivers in rural area of Andra Pradesh. Nat J Community Med. 2015; 6(4):566-570.

16. Sangaleti CT, Trincaus MR, Baratieri T, Zarowy K, Ladika MB, Menon MU, et al. Prevalence of cardiovascular risk factors among truck drivers in the South of Brazil. BMC Public Health. 2014; 14:1063.

17. Shin SY, Lee CG, Song HS, Kim SH, Lee HS, Jung MS, et al. Cardiovascular Disease Risk of Bus Drivers in a City of Korea. Ann Occup Environ Med. 2013; 25(1):34

18. Siu SC, Wong KW, Lee KF, Lo YY, Wong CK, Chan AK, et al. Prevalence of undiagnosed diabetes mellitus and cardiovascular risk factors in Hong Kong professional drivers. Diabetes Res Clin Pract. 2012; 96(1):60-67

19. Camargo-Escobar FL, Gómez-Herrera OL, López-Hurtado MX. Riesgo cardiovascular en conductores de buses de transporte público urbano en Santiago de Cali, Colombia. Rev Colomb Salud Ocup. 2013; 3(3):18-22.

20. Hirata RP, Sampaio LM, Leitao Filho FS, Braghiroli A, Balbi B, Romano S, et al. General Characteristics and Risk Factors of Cardiovascular Disease among interstate Bus Drivers. Sci World J. 2012; 2012:216702.

21. Hinestroza JF, Giraldo JC. Riesgo cardiovascular en conductores de servicio público intermunicipal, 2008. Rev Méd Risaralda. 2009; 15(2):13-21.

22. Elshatarat RA, Burgel BJ. Cardiovascular Risk Factors of Taxi Drivers. J Urban Health. 2016; 93(3):589-606 